\title{
RNF13: a novel RING-type ubiquitin ligase over-expressed in pancreatic cancer
}

\author{
Qiang Zhang ${ }^{1, *}$, Yunxiao Meng ${ }^{2, *}$, Lei Zhang ${ }^{1, *}$, Jie Chen $^{2}$, Dahai Zhu ${ }^{1}$ \\ ${ }^{1}$ National Laboratory of Medical Molecular Biology, Institute of Basic Medical Sciences, Chinese Academy of Medical Sciences, \\ School of Basic Medicine, Peking Union Medical College, Tsinghua University, 5 Dong Dan San Tiao, Beijing 100005, China; ${ }^{2}$ De- \\ partment of Pathology, Peking Union Medical College Hospital, Chinese Academy of Medical Sciences and Peking Union Medical \\ College, Tsinghua University, 1 Shuai Fu Yuan Hu Tong, Beijing 100730, China
}

Protein ubiquitination by E3 ubiquitin ligases plays an important role in cancer development. In this study, we provide experimental evidence that a RING-finger-containing protein RNF13 is an ER/Golgi membrane-associated E3 ubiquitin ligase and its RING finger domain is required for the ubiquitin ligase activity. Immunohistochemical analysis of pancreatic ductal adenocarcinoma (PDAC) and paracancerous normal tissues from 72 patients documented RNF13 over-expression in 30 tumor samples $(41.7 \%, 30 / 72)$, and its expression was significantly associated with histological grading $(P=\mathbf{0 . 0 2 4})$. In addition, RNF13 was detected in precancerous lesions: tubular complexes in chronic pancreatitis (CP) and pancreatic intraepithelial neoplasia (PanIN) (79.3\%, 23/29 and 62.8\%, 22/35, respectively). Moreover, RNF13 staining was significantly correlated with Tenascin-C expression $(P=0.004)$ in PDAC samples, further supporting the role of RNF13 in cancer progression. Over-expression of wild type but not RING domain-mutant RNF13 in pancreatic MiaPaca-2 cancer cells increased invasive potential and gelatinolytic activity by matrix metalloproteinase-9. Taken together, these findings reveal that RNF13 is a novel E3 ubiquitin ligase involved in pancreatic carcinogenesis; ubiquitin-mediated modification of proteins by RNF13 may participate in pancreatic cancer development.

Keywords: RNF13, ubiquitin ligase, pancreatic cancer, histological grading, PanINs, invasion

Cell Research (2009) 19:348-357. doi: 10.1038/cr.2008.285; published online 16 September 2008

\section{Introduction}

Many RING-finger-containing monomeric proteins and multi-subunit complexes possess ubiquitin ligase activity and play a crucial role in degradation and trafficking of regulatory proteins; several have been reported to be involved in tumorigenesis and cancer metastasis [1]. For example, RNF11 enhances transforming growth factor- $\beta$ signaling and suppresses the progression of breast cancer by binding and degrading Smurf2 [2,3]. Breast cancer-associated gene 2 (BCA2) is an E3 with a RING domain that is over-expressed in invasive breast cancer and positively correlates with estrogen receptor expression and survival,

\footnotetext{
*These authors contributed equally to this work.

Correspondence: Jie Chen ${ }^{\mathrm{a}}$, Dahai Zhu ${ }^{\mathrm{b}}$

${ }^{a}$ Tel/Fax: +86-10-6529-5490

E-mail: xhblk@163.com

${ }^{\mathrm{b}}$ Tel: +86-10-6529-6949; Fax: +86-10-6510-5083

E-mail: dhzhu@pumc.edu.cn

Received 8 July 2008; accepted 7 August 2008; published online 16 September 2008
}

but negatively with lymph node metastasis [4]. Recently ubiquitin ligase SCF- $\beta$ - TrCP1 has been shown to be a critical determinant for Smad4 protein degradation in pancreatic cancer cells, and the somatic homozygous H460R mutation in FBXW7, a subunit of an SCF-type ubiquitin ligase complex associated with Cyclin E over-expression, was observed in a subset of pancreatic cancers $[5,6]$. A hallmark of pancreatic cancer is its extensive local invasion and early systemic dissemination. The vast majority of pancreatic cancer patients present with advanced and incurable disease [7]. Therefore, identification and characterization of novel genes involved in pancreatic cancer development from precancerous lesions, such as pancreatic intraepithelial neoplasia (PanIN), would be a logical requirement for the prevention and effective treatment of pancreatic cancer [8].

RNF13 or C-RZF (GenBank accession number NM 205355.1) was initially identified in chicken embryo brain by way of its expression being stimulated by the substrate adhesion molecule Cytotactin/Tenascin-C [9]. The latter is up-regulated in acute and chronic pancreatitis (CP), PanINs 
and pancreatic cancer, and the increased expression of Tenascin-C is correlated with pancreatic cancer differentiation [10]. Expression level, splicing variants and distribution of Tenascin-C were also found to be associated with the progression of several cancers, especially breast cancer and brain tumors [11-13]. In addition, the human RNF13 gene (GenBank accession number NM_007282.1) is localized at chromosome $3 \mathrm{q} 25.1$, a site suggested to harbor genes involved in pancreatic cancer development [14]. Therefore, it is conceivable to investigate the potential role of RNF13 in the pathogenesis of human pancreatic cancer.

In the present study, we provide experimental evidence that RNF13 is a trans-membrane RING-type E3 ubiquitin ligase highly expressed in pancreatic ductal adenocarcinoma (PDAC) and precancerous lesions. A significant association between RNF13 expression and histological grade of PDAC or the expression of Tenascin-C was also observed. Finally, ubiquitin ligase activity of RNF13 was found to be involved in pancreatic cancer cell invasive potential in vitro.

\section{Results}

RNF13 encodes a membrane glycoprotein associated with ER/Golgi

The RNF13 gene is located on human chromosome $3 \mathrm{q} 25.1$ and encodes a protein product of 381 amino acids. The RNF13 protein (NP_009213.1) contains several predicted functional domains, including a protease-associated (PA) domain, a RING-H2 type RING finger domain, a signal peptide (SP), a nuclear localization signal (NLS) and a transmembrane (TM) region (Figure 1A). These features suggest that RNF13 is a member of GRAIL (Gene Related to Anergy in Lymphocytes) paralogs, which are characterized by PA-TM-RING domains [15]. Analysis of RNF13 expression in multiple human tissues by Western blotting showed that RNF13 is expressed as a $60 \mathrm{kDa}$ protein (Figure 1B). The sub-cellular localization of RNF13 was determined in MiaPaca-2 pancreatic cancer cells transfected with the Myc-tagged RNF13 expression vector. Figure $1 \mathrm{C}$ shows that the RNF13 protein is predominantly localized in the cytoplasm and co-localizes with the ER protein Bip/GRP78 and Golgi antigen, but not with the early endosome resident EEA1; this behavior is similar to that of other members of the GRAIL family, such as GRAIL and RING105 [16, 17]. Furthermore, RNF13 was found to be enriched in the nonionic detergent Triton $\mathrm{X}-114$ phase, supporting its association with membranes (Figure 1D). Pretreatment of RNF13-transfected cells with tunicamycin (an $\mathrm{N}$-glycosylation inhibitor) and extraction of total cellular proteins with $N$-glycosidase F demonstrated a mobility shift of the RNF13 protein from 60 to $50 \mathrm{kDa}$
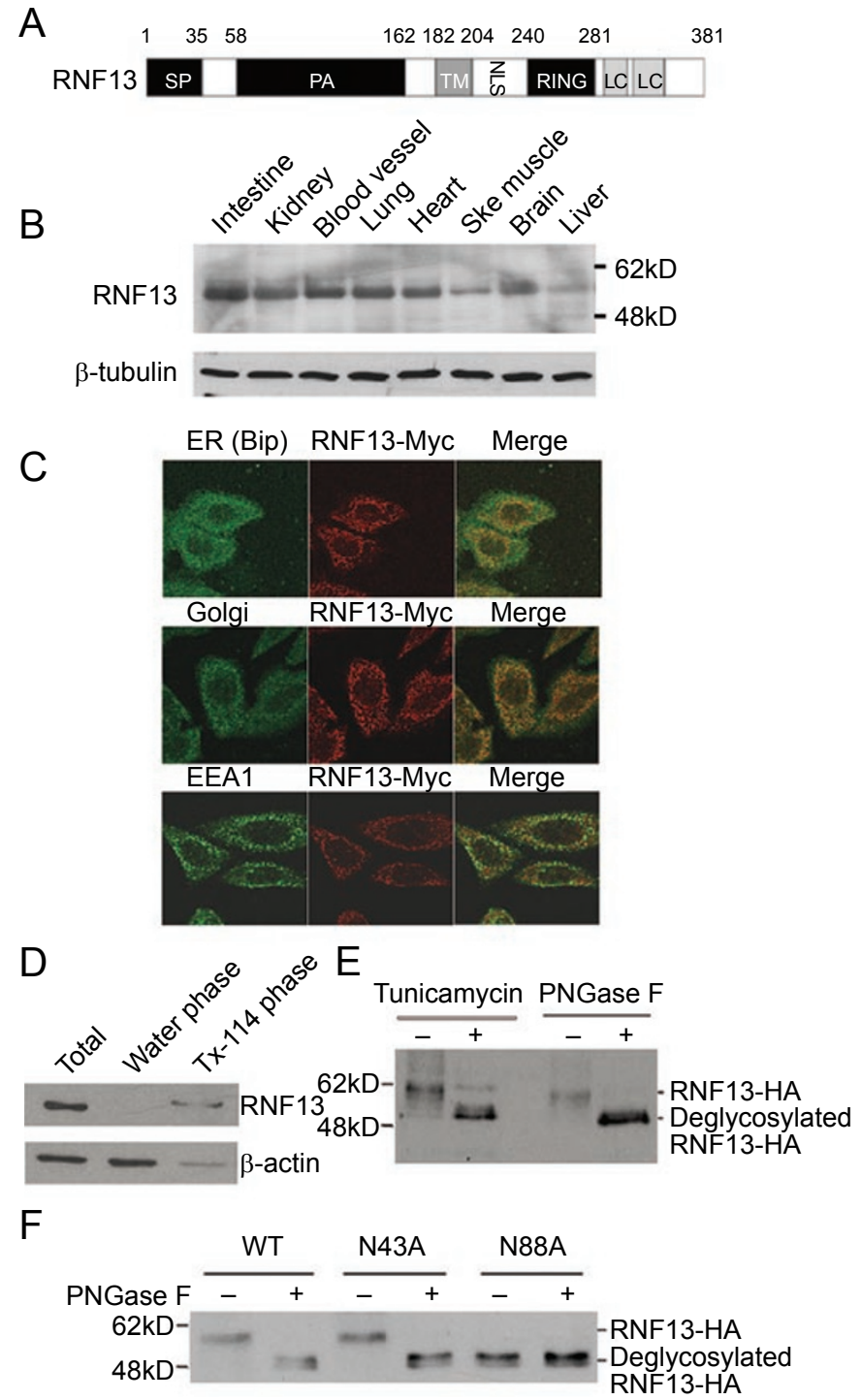

Figure 1 RNF13 protein sequence analysis, localization and glycosylation assay. (A) SP: signal peptide; PA: protease-associated domain; TM: transmembrane region; RING: RING finger domain; NLS: nuclear localization signal; LC: low complexity. (B) Western blot analysis of RNF13 protein in multiple human tissues using anti-RNF13 antibody. (C) MiaPaca-2 cells transiently expressing RNF13 with a C-terminal Myc epitope tag were used for immunofluorescence analysis of subcellular localization of RNF13. MiaPaca-2 cells co-stained for RNF13-Myc and anti-Bip (ER), anti-Golgi (Golgi), or anti-EEA1 (early endosomes). (D) Phase partition of RNF13 in Triton X-114. Total proteins were extracted with $1 \%$ Triton $\mathrm{X}-114$ directly from isolated cells. $\beta$-actin was used as control. (E) COS-7 cells transfected with RNF13-HA were treated with tunicamycin $(1.5 \mu \mathrm{g} / \mathrm{mL})$ for $24 \mathrm{~h}$. $N$-glycosidase $F$ was used for treatment of $5 \mu \mathrm{g}$ of total proteins expressing RNF13-HA from COS-7 cells. Protein samples were subjected to immunoblot with anti-HA antibody. (F) Wild-type RNF13 and its N43A and N88A mutants expressed in COS-7 cells were treated in the presence or absence of $\mathrm{N}$-glycosidase $\mathrm{F}$ and detected with anti-HA antibody. 
(Figure 1E), suggesting that RNF13 is an $N$-glycosylated protein. To identify the $N$-glycosylated sites in RNF13 protein, two mutants (RNF13 N43A and N88A) were created at two predicted glycosylation sites (N43 and N88). RNF13 N88A displayed a lower molecular weight, whereas the RNF13 N43A mutant shifted together with wild-type RNF13 (Figure 1F). These results suggest that RNF13 is glycosylated at N88.

RNF13 is a novel E3 ubiquitin ligase

Sequence alignment analysis showed that the RING finger domain of RNF13 shares significant homology with several known E3 ligases (Figure 2A), suggesting that RNF13 could be an E3 ubiquitin ligase. To test this possibility we performed an auto-ubiquitination assay, which is a specific test for demonstration of a RING-finger-domain-containing E3 ubiquitin ligase [18, 19]. Recombinant RNF13 (207-374) containing the entire RING domain exhibited ubiquitin ligase activity. This was activated by $\mathrm{UbcH} 5 \mathrm{a}$ and $\mathrm{UbcH} 5 \mathrm{c}$ in an ATP- and E1-dependent manner (Figure 2B); all components were required for the polyubiquitination of RNF13 (Figure 2C). To further confirm

A

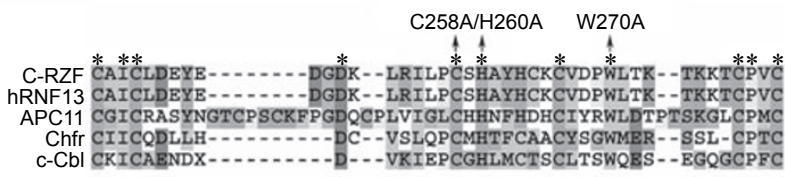

B
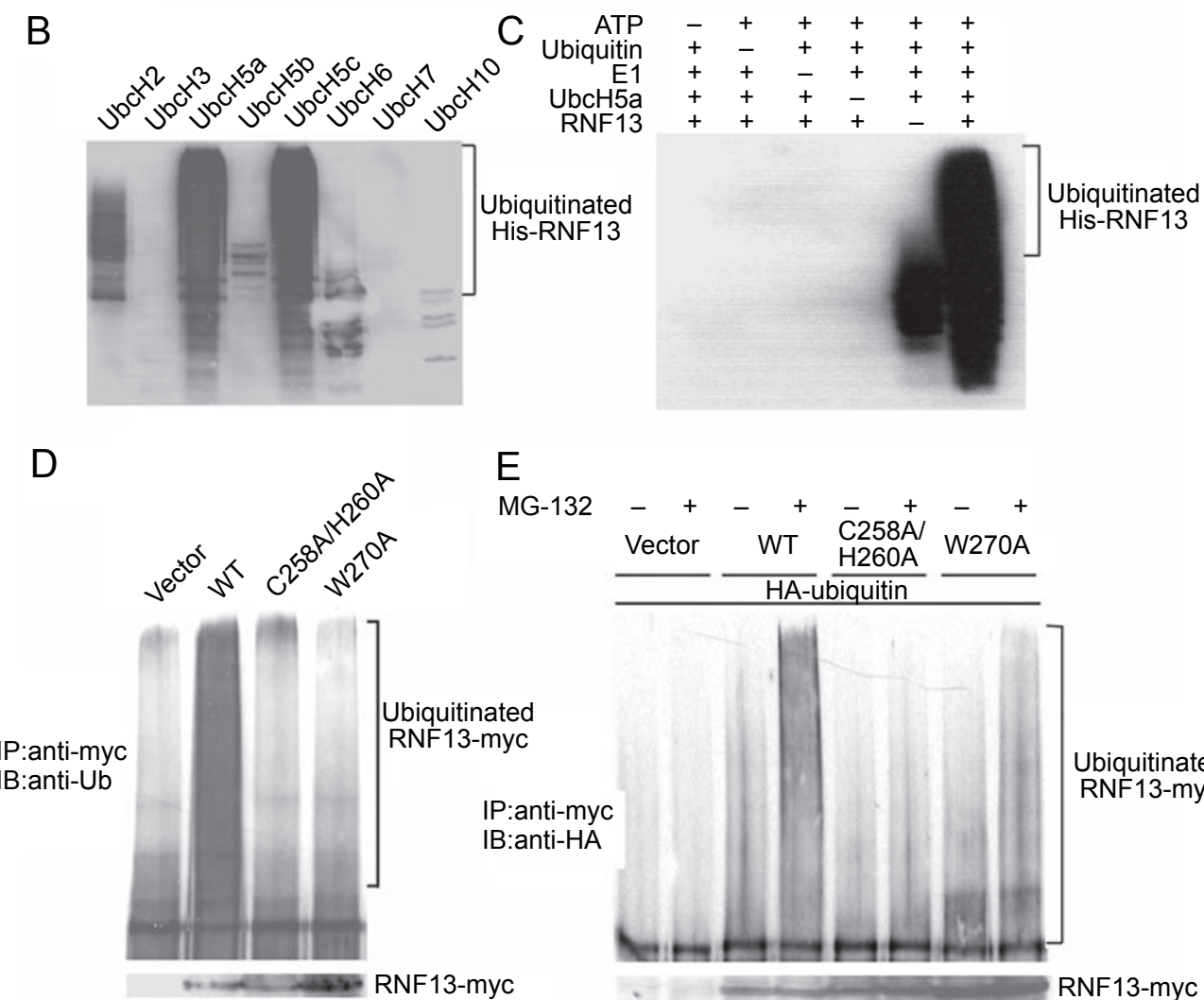

$E$ MG-132

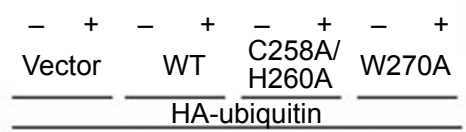

Figure 2 RNF13 protein demonstrates E3 ligase activity. (A) Alignments of the RING finger domain from human RNF13, C-RZF, APC11, Chfr, C-Cbl. (B) Ubiquitination assay was performed using purified recombinant RNF13 (C-terminal 207-374 amino acids) along with ATP, ubiquitin, E1 and various recombinant human E2 enzymes. The formation of the ubiquitin-RNF13 conjugates was analyzed by immunoblotting with anti-ubiquitin antibody. (C) Determination of components necessary for RNF13-mediated ubiquitination. One component was omitted in each reaction as follows: Lane 1, ATP. Lane 2, ubiquitin. Lane 3, E1. Lane 4, E2 UbcH5a. Lane 5, RNF13. (D) The immunoprecipitates from COS-7 cells, transfected with wild-type and RING mutant RNF13, were incubated with ubiquitin, E1 and UbcH5a and assayed for E3 ligase activity with anti-ubiquitin antibody. (E) COS-7 cells were co-transfected with HA-ubiquitin and Myc-RNF13, or Myc-RNF13 (C258A/H260A), or Myc-RNF13 (W270A), or control vector. After transfection, the cells were treated with (+) or without $(-)$ MG-132 (10 $\mu \mathrm{M})$ for $4 \mathrm{~h}$. Immunoprecipitates were analyzed with anti-HA antibody. 
the requirement for the RING finger domain for RNF13 ubiquitin ligase activity, we carried out the ubiquitination assay with the wild-type RNF13 and two RNF13 RING finger mutants, C258A/H260A and W270A. As indicated in Figure 2D, both mutants almost completely lost their E3 ubiquitin ligase activity. In addition, we observed that RNF13 exerted E3 ubiquitin ligase activity in vivo, especially in the presence of the proteasome inhibitor MG-132. As indicated in Figure 2E, one of the RNF13 RING finger domain mutants, C258A/H260A, completely lost its E3 ligase activity while the other mutant W270A maintained little E3 ligase activity. In summary, these data confirm that RNF13 is a bona fide RING-type E3 ubiquitin ligase.

\section{RNF13 expression is associated with histological grading} in patients with $P D A C$

It has been reported that RNF13 expression is up-regu- lated by the cancer-related extracellular protein TenascinC [9]; this prompted the study of RNF13 role in cancer development through the analysis of its expression pattern in 72 PDAC patients. RNF13 expression was analyzed by immunohistochemistry with the commercially available anti-RNF13 polyclonal antibody (Abcam 4288). We first tested the sensitivity and specificity of the antibody (Supplementary information, Figure S1). Then RNF13 protein expression was examined in normal pancreas tissue; the results indicated that RNF13 is detectable only in islets but not in pancreatic ducts and acini (Figure 3A and $3 \mathrm{G})$. Interestingly, RNF13 was found to be expressed in pancreatic ducts and acini in PDAC samples, suggesting that RNF13 expression is likely associated with pancreatic cancer development. To further verify this association, RNF13 expression was examined in samples from the 72 PDAC patients; intensity of staining was scored as de-

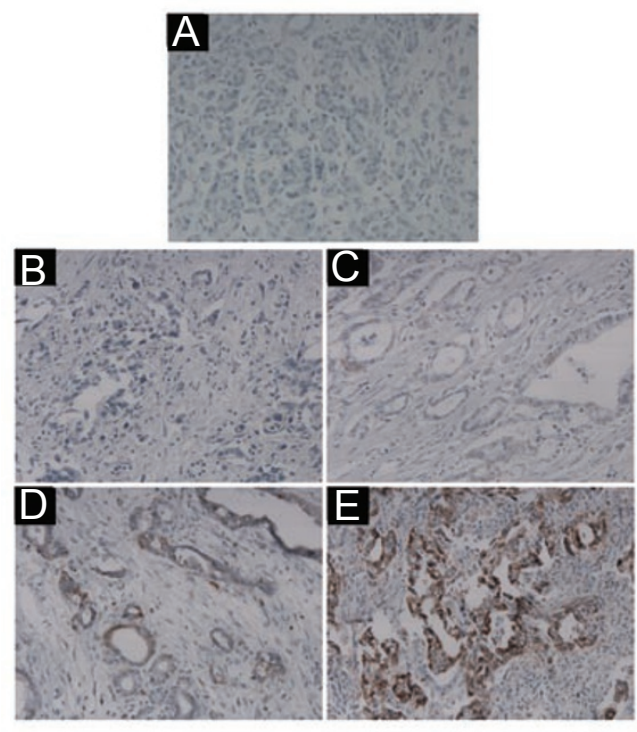

G

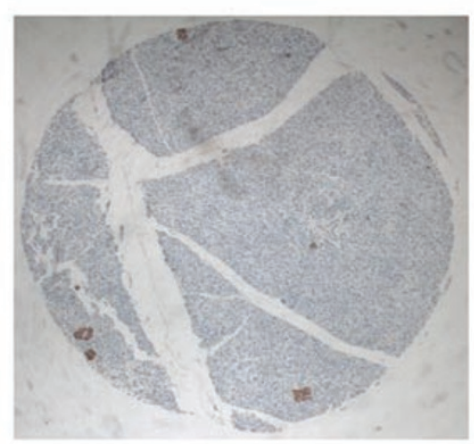

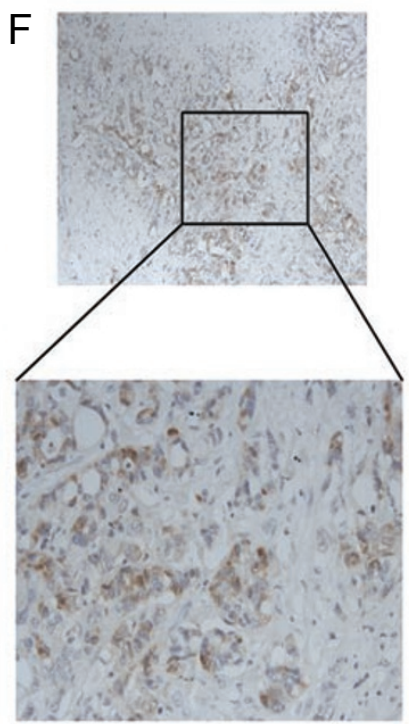

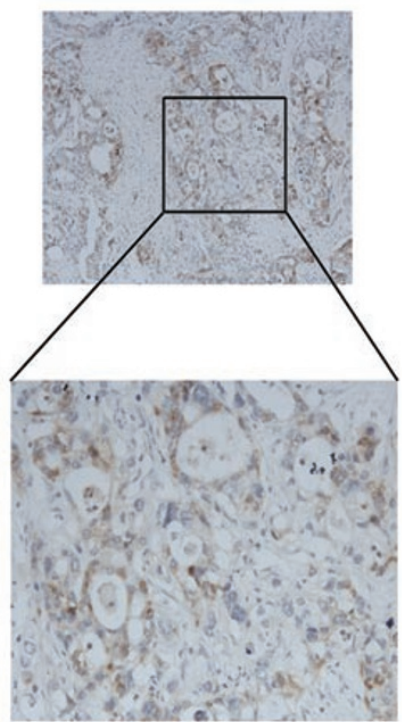

Pancreatic cancer

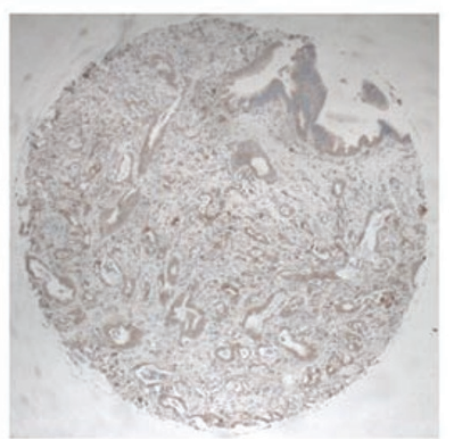

Figure 3 Different scores of RNF13 staining and representation of RNF13 staining in PDAC cases and tissue array. (A) Normal pancreas tissue. Scoring of the immunoreactivity of RNF13 was estimated by the percentage of labeled tumor cells. (B-E) Reflection of the staining scores of samples from 0 to +++ , respectively ( $\times 40$ magnification). (F) Two representative clinical samples of pancreatic ductal adenocarcinoma ( $\times 40$ magnification and $\times 100$ magnification). (G) Normal pancreatic tissue and PDAC sample from PDAC tissue array. 
scribed in Materials and Methods. Figure 3B-3E provides representative results with a scoring scale of 0 to $+1+$. Thirty PDAC samples were found to be positive $(41.7 \%, 30 / 72)$ as exemplified by Figure 3F. This observation was further validated in pancreatic cancer tissue arrays containing an independent set of 79 PDAC samples; 30 out of 79 samples stained positive for RNF13 (37.97\%, 30/79, Figure 3G). Collectively, these results indicate that the E3 ubiquitin ligase RNF13 is over-expressed in human PDAC.

To determine whether RNF13 expression is correlated with clinico-pathological parameters, the relationship between RNF13 expression, histological grading and clinical stage was analyzed in the 72 PDAC patients. We found that elevated expression of RNF13 was significantly correlated with histological grading $(P=0.024)$ but not with pathologic stage, lymph node metastasis and depth of invasion (Table 1). Positive staining of RNF13 was observed predominantly in well-differentiated tumors (G1, 17/29), while negative staining of RNF13 was found in poorlydifferentiated tumors (G3, 18/23), suggesting that RNF13 expression is associated with well-differentiated PDAC.

RNF13 is expressed in the tubular complexes in CP and PanIN

We then investigated the role of RNF13 in early-stage pancreatic cancer by analyzing RNF13 expression in precancerous lesions including tubular complexes in $\mathrm{CP}$ and PanIN. In CP adjacent to PDAC, 23 out of 29 (79.3\%) samples exhibited positive staining of RNF13 in tubular
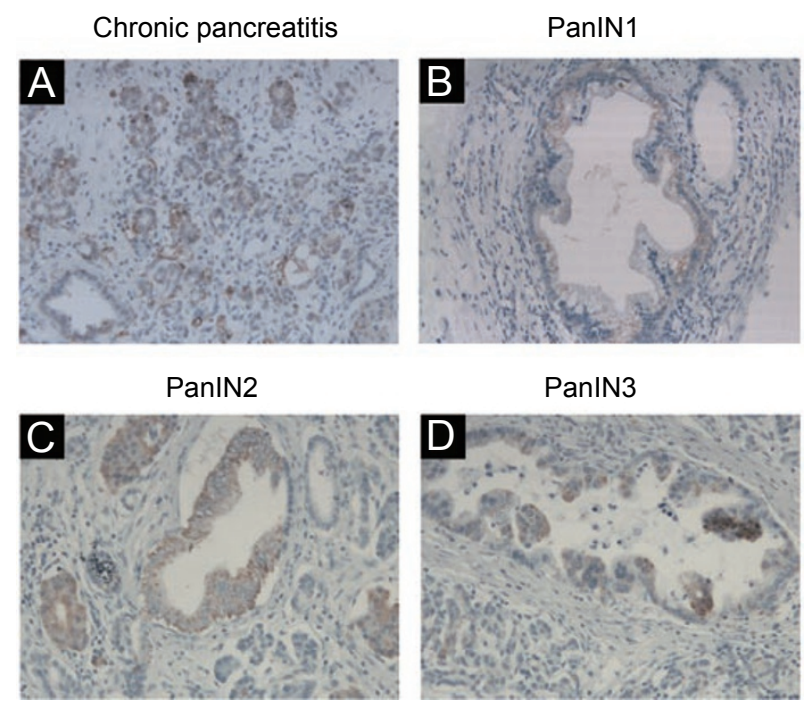

Figure 4 Expression of RNF13 in the tubular complexes of CP and PanINs. (A) Cells of tubular complexes in CP in RNF13-positive specimen ( $\times 100$ magnification). (B-D) RNF13-positive staining in representative clinical specimens of PanIN1, PanIN2 and PanIN3 ( $\times 100$ magnification).
Table 1 Association between RNF13 expression and comparison of groups for clinico-pathologic variables of 72 PDAC patients

\begin{tabular}{|c|c|c|c|c|}
\hline \multirow[t]{2}{*}{ Variable $\mathrm{N}$} & \multirow{2}{*}{ No. of patients } & \multicolumn{2}{|c|}{ RNF13 expression } & \multirow{2}{*}{$P^{*}$} \\
\hline & & \multicolumn{2}{|c|}{ Positive $(n=30)$ Negative $(n=42)$} & \\
\hline \multicolumn{4}{|l|}{ Age } & 0.431 \\
\hline$<65$ & 52 & 20 & 32 & \\
\hline$\geqslant 65$ & 20 & 10 & 10 & \\
\hline \multicolumn{4}{|l|}{ Gender } & 0.315 \\
\hline Male & 46 & 17 & 29 & \\
\hline Female & 26 & 13 & 13 & \\
\hline \multicolumn{4}{|l|}{ Node Status } & 0.469 \\
\hline Negative & 28 & 10 & 18 & \\
\hline Positive & 44 & 20 & 24 & \\
\hline \multicolumn{4}{|c|}{ Depth of invasion } & 0.265 \\
\hline T1 & 2 & 2 & 0 & \\
\hline $\mathrm{T} 2$ & 16 & 8 & 8 & \\
\hline $\mathrm{T} 3$ & 49 & 19 & 30 & \\
\hline $\mathrm{T} 4$ & 5 & 1 & 4 & \\
\hline \multicolumn{4}{|c|}{ Pathologic stage } & 0.358 \\
\hline I & 6 & 3 & 3 & \\
\hline II & 16 & 7 & 9 & \\
\hline III & 42 & 19 & 23 & \\
\hline IV & 8 & 1 & 7 & \\
\hline \multicolumn{4}{|l|}{ Histology } & $0.024 \dagger$ \\
\hline G1 & 29 & 17 & 12 & \\
\hline $\mathrm{G} 2$ & 20 & 8 & 12 & \\
\hline G3 & 23 & 5 & 18 & \\
\hline
\end{tabular}

* Fisher's exact test was used for analysis

$\dagger$ Statistically different $(P<0.05)$

complexes (ductal-like cells, Figure 4A). Twenty-two of 35 PanIN cases (62.8\%) were RNF13 positive (Figure 4B-4D). As reported, PanIN can be classified into three main different stages $(1,2,3)$ based on epithelial cells' hyperplasia and morphology; in this study, 9 PanIN-1, 13 PanIN-2 and 13 PanIN-3 samples were included. RNF13 expression was observed in 5/9 (55.6\%) cases in PanIN-1, 7/13 (53.8\%) in PanIN-2 and 10/13 (76.9\%) in PanIN-3 (Figure 4B-4D). No significant difference in RNF13-positive staining of ductal cells was found among the three different PanIN lesions $(P=0.129)$. Since PanIN has been considered a precursor of PDAC, these results suggest that RNF13 expression is associated with the early stages of pancreatic cancer development.

\section{RNF13 and Tenascin-C are co-expressed in PDAC}

$\mathrm{RNF} 13$ expression is up-regulated by Tenascin-C [9], an extracellular matrix glycoprotein that has been proposed as a tumor marker with prognostic significance in many human cancers [20]. This prompted us to investigate a 
potential correlation between RNF13 and Tenascin-C expression in PDAC. Tenascin-C expression was detected in 35 of 72 (48.6\%) tumor samples. Immunological staining indicated that Tenascin-C expression is concentrated in the tumor stroma (Figure 5A) while consecutive sections stained strongly positive for RNF13 in the cells (Figure 5B). Of 30 RNF13-positive samples 21/30 (70\%) co-expressed Tenascin-C, and of 42 RNF13-negative samples 28/42 $(66.7 \%)$ were negative for Tenascin-C (odds ratio (OR), 4.67). The expression of the two proteins was significantly correlated in PDAC samples $(P=0.004)$ (Table 2$)$.

\section{RNF13 promotes invasion in pancreatic cancer cells}

Based on the above observations, we then investigated possible molecular functions of RNF13 in pancreatic cancer cells. Cell proliferation and invasion were examined in the MiaPaca-2 pancreatic cancer cell line, which stably over-expresses either wild-type RNF13 (MiaP2-RNF13 WT No. 4, and No. 6) or RNF13 RING domain mutant C258A/H260A (MiaP2-RNF13 Mt. No. 1 and No. 2), and two mock-transfected clones (MiaP2-Vec1 and MiaP2-Vec2) (Figure 6A). The cell proliferation assay indicated that there was no significant difference in growth rates and cell viability among the three groups (Figure 6B). We next examined whether RNF13 could affect the invasive potential of pancreatic cancer cells by Matrigel invasion assay; MiaPaca- 2 cells overexpressing wild-type RNF13 exhibited enhanced invasive ability, whereas the ubiquitin ligase-deficient RNF13 mutant failed to promote cell invasion (Figure 6C). To test whether RNF13 expression in MiaPaca-2 cells affects the activity of matrix metalloproteases, key enzymes for degradation of extracellular matrix, we performed a gelatin zymography assay; increased MMP-9 (gelatinase B) activity was only detected in cells carrying the wild-type RNF13, but not in those expressing the RNF13 RING mutant or control vector. However, all cell lines from the three groups exhibited similar MMP-2 (gelatinase A) activity (Figure 6D and 6E). Hence, our data demonstrated that RNF13 might play a functional role in enhancing pancreatic cancer cell invasion

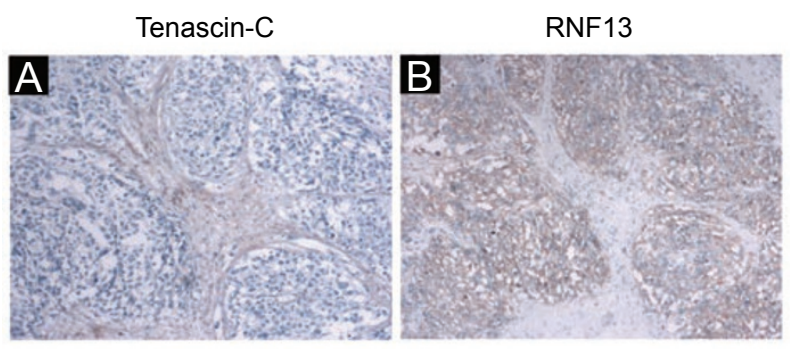

Figure 5 Comparison of representative PDAC for RNF13 and Tenascin- $C$ staining. The consecutive sections were stained for Tenascin-C (A) and RNF13 (B) and viewed at $\times 40$ magnification.

Table 2 Correlation of RNF13 and Tenascin-C expression in 72 PDAC patients

$\frac{\text { RNF13 expression }}{\text { Positive }(n=30) \quad \text { Negative }(n=42)} P^{*}$

Tenascin-C expression

Positive $(n=35) \quad 21 \quad 14$

Negative $(n=37) \quad 98$

* Fisher's exact test was used for analysis

$\uparrow$ Statistically different $(P<0.05)$

in vitro by promoting metalloprotease function.

\section{Discussion}

RNF13 was initially identified as a Tenascin-C-stimulated factor; at the same time, elevated Tenascin-C expression was observed in precancerous lesions and correlated with differentiation of PDAC $[9,10,21]$. Thus, we investigated the pathologic/clinical relevance of RNF13 expression in PDAC; this study indicates that RNF13 is a novel tumorassociated E3 ubiquitin ligase that may indeed play a role in the early phase of PDAC. RNF13 was over-expressed in PDAC samples from individual patients and in an independent set of samples from archival tissue microarray compared to normal tissues. Furthermore, RNF13 expression correlated significantly with expression of the cancer-related Tenascin-C in the same PDAC samples, and over-expression of RNF13 in pancreatic cancer cells enhanced invasiveness and MMP-9 activity. Finally, microarray analysis of RNF13 expression also demonstrated higher level of RNF13 mRNA in some basal cell carcinoma, epithelial ovarian carcinoma and melanoma (personal communication from Francesco M Marincola, Department of Transfusion Medicine, NIH).

RNF13 contains an N-terminal PA domain and a Cterminal RING finger domain, which is characteristic of GRAIL paralogs [15]. The PA domain, a motif found in plant vacuolar sorting proteins, transferrin receptor and different protease superfamilies, could serve as a proteininteracting domain $[22,23]$. The RING finger domain, a unique feature of RING-type E3 ubiquitin ligase, plays an important role in ubiquitin-mediated protein modification by mediating the direct transfer of E2-bound ubiquitin to substrates without thioester bond formation [24]. We have shown that RNF13 is a RING-type ubiquitin ligase and that the RING finger domain of RNF13 is necessary and sufficient for its ability to catalyze ubiquitination. Like most RING E3 ligases showing short half-life because of auto-ubiquitination, the stability of wild-type but not RING mutant RNF13 is regulated by the proteasome (data not shown). Under normal circumstances, the protein level 


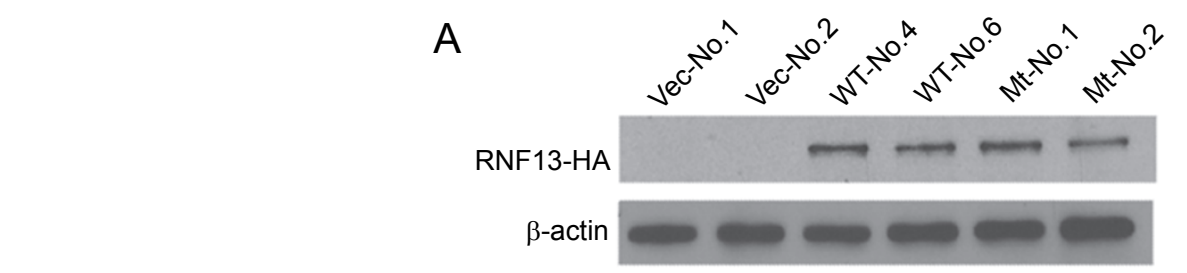

B

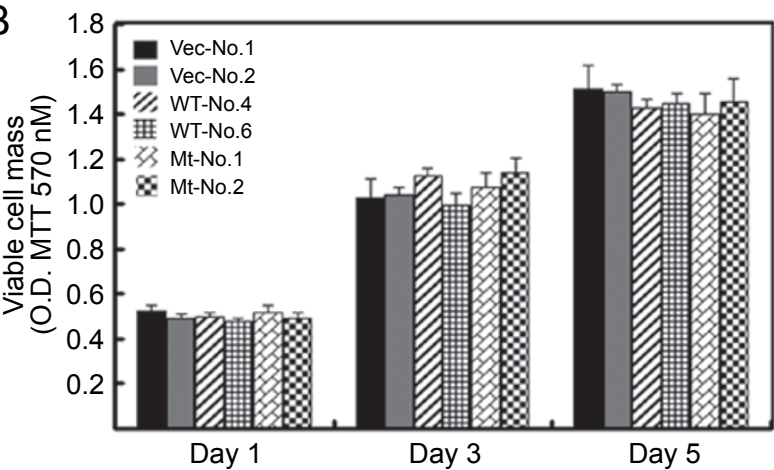

D

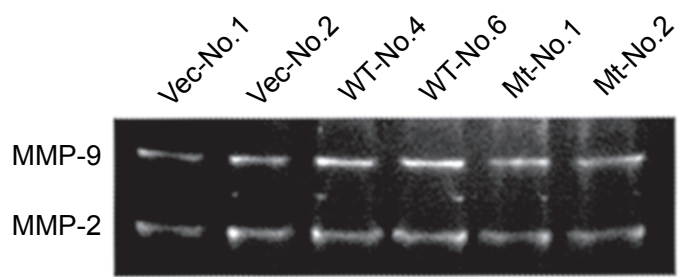

C

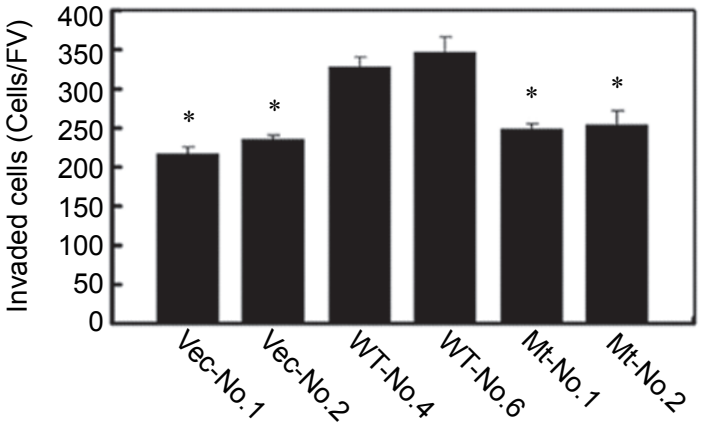

E

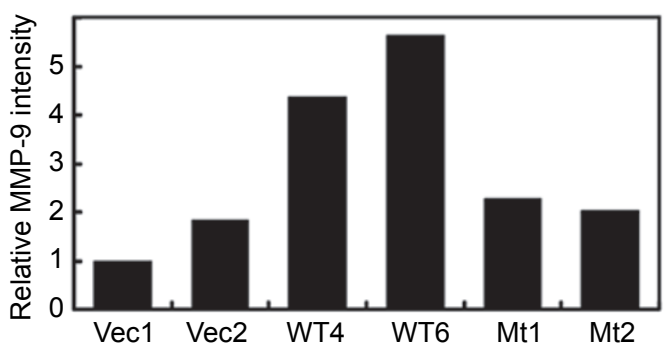

Figure 6 Effect of RNF13 on proliferation and invasion in pancreatic cancer cells. (A) Western blotting analysis of wild-type and RING mutant RNF13 stable expression in MiaPaca-2 stable clones. Two vector control clones (MiaP2-vector No. 1 and No. 2), wild-type RNF13 stable clones (MiaP2-RNF13 WT No. 4 and No. 6) and RING mutant RNF13 stable clones (MiaP2-RNF13 Mt No. 1 and No. 2). (B) Comparison of growth of MiaP2-RNF13 WT, MiaP2-RNF13 Mt and MiaP2-Vector cells at different days (day 1, 3, 5) with the same seeding number in MTT proliferation assay. (C) Quantitative evaluation of invasion of the RNF13 transfectants (WT and RING mutant) and vector transfectants in the Matrigel invasion assay. Tumor cell invasion was quantified as the mean cell number of invaded cells in five fields of view per filter (cells/FV). Columns, mean of three independent experiments; bars, \pm SD. (D) Cultured media of mock transfectants, wild-type and RING mutant RNF13 transfectants were condensed and MMP-2 and MMP-9 activities was assayed by gelatin zymography. (E) Relative gelatinolytic band MMP-9 intensities were quantified by densitometry using Bio-Rad Quantity One software.

of RNF13 may be strictly regulated by its expression, auto-ubiquitination and specific ubiquitin protease, which may be crucial for the maintenance of cell homeostasis. Dysfunction of E3 ubiquitin ligases is implicated in cancer development, because of their central role in promoting the ubiquitination of proteins that are essential for a variety of cellular functions. Many RING-type E3 ligases could be oncoproteins or tumor suppressors because their deregulation has been shown to be associated with human carcinogenesis. For instance, the G allele of MDM2 promoter $309 \mathrm{~T} / \mathrm{G}$ polymorphism, which is associated with higher MDM2 mRNA and protein levels, leads to a 2- to 3 -fold increased risk of developing pancreatic cancer and to a worse prognosis in patients who develop the disease [25]. In this study, we observed that elevated expression of
RNF13 in PDAC samples was significantly correlated with histological grading $(P=0.024)$ and often detected in welldifferentiated samples (17/29), providing additional new information supporting a functional role of E3 ubiquitin ligases in pancreatic cancer development.

RNF13 expression was associated with the expression of Tenascin-C in PDAC ( $P=0.004$; OR, 4.67), suggesting that RNF13 may participate coordinately with Tenascin-C in mediating carcinogenesis. Although in the 72 PDAC samples examined here the ratio of Tenascin-C positive staining was lower than reported by others, co-expression of RNF13 and Tenascin-C supports their combined role in cancer development [10]. Stromal cells such as carcinomaassociated fibroblasts (CAFs) can secrete Tenascin-C, which stimulates Wnt/ $\beta$-catenin, MAP kinase and EGFR 
signaling at different stages of cancer development in different cancer cell types [26, 27]. RNF13 may participate in Tenascin-C-mediated signaling and possibly it might mediate the function of Tenascin-C.

The observation that RNF13 is highly expressed in precancerous lesions such as PanINs $(62.8 \%)$ and in tubular complexes $(79.3 \%)$ in $\mathrm{CP}$ is of further significance. Detection and treatment of early, non-invasive PanIN can have a major impact on pancreatic cancer survival. PanINs arise in the smaller pancreatic ducts and are classified morphologically into three grades, according to nuclear polarity, nuclear size (pleomorphism) and hyper-chromatic staining [28]. Despite complex interactions between tumor cells and PanINs, histological and molecular evidence suggests that PanINs can gradually progress to PDACbearing genetic traits such as K-ras mutation, Cyclin D1 over-expression and loss of p16 expression [29, 30]. Since $\mathrm{CP}$ is also an independent risk factor for the development of PDAC [31], the high proportion of RNF13 expression in CP and PanINs suggests that RNF13 might be involved in the earliest stages of pathogenesis of PDAC and could potentially be used as a biomarker for diagnosis of PDAC at a non-invasive stage.

As shown in Figure 1C, RNF13 is localized in the membrane of ER and Golgi, indicating that RNF13 is likely a type I TM protein: the N-terminal PA domain would be internal toward the lumen and the $\mathrm{C}$ terminus RING finger domain would face the cytosol. The integral membrane localization of RNF13 implicates that both the cytoplasmic and membrane-associated proteins could be ubiquitinated by RNF13. The ectopic expression of RNF13 in transformed pancreatic cancer cells increased the activity of extra-cellular MMP-9 (gelatinase B) that contains a fibronectin-like sequence and plays a critical role in tumor invasion and metastasis [32]. In view of the ability of wild-type but not RING mutant RNF13 to function as an E3 ligase and promote pancreatic cancer cell invasion, our results suggest that the increase of MMP-9 activity is likely due to protein ubiquitination by RNF13. The increased invasive activity of RNF13 transfectants suggests that RNF13 might play a functional role in the later stages of cancer development in vivo. In addition, our data derived from the study of patients with PDAC demonstrated high frequency of RNF13 expression in precancerous lesions and an association with well-differentiated tumors, pointing to the possibility that RNF13 might stimulate proliferation and enhance tumorigenesis. Taken together, our findings imply that RNF13, as an E3 ubiquitin ligase, might mediate pancreatic cancer initiation, progression and invasion at multiple stages through ubiquitination and consequent modification of various substrates. Therefore, further identification of RNF13-mediated ubiquitination substrate(s) and characterization of its role in mediating the actions of Tenascin-C and other extracellular matrix proteins will not only shed light on the molecular mechanism of RNF13 function in cancer development but also provide a molecular basis for the use of RNF13 as a diagnostic and therapeutic target in cancer.

\section{Materials and Methods}

\section{Cell lines and antibodies}

MiaPaca-2, COS-7 cells were obtained from the American Type Culture Collection. Anti-human RNF13 antibody was purchased from Abcam (abcam 4288) (Abcam Company Research, UK). The monoclonal antibody anti-Tenascin-C (clone BC-24) and HA antibody were obtained from Sigma (St. Louis, MO). The endoplasmic reticulum protein BiP/GRP78 antibody and the early endosome protein EEA1 antibody were from BD transduction Laboratories (Palo Alto, CA). The anti-Golgi Apparatus mAb (AE-6) was from Calbiochem (LaJolla, CA). The anti-Ubiquitin (P4D1), anti-Myc (9E10), anti- $\beta$-Actin and anti- $\beta$-Tubulin antibodies were purchased from Santa Cruz Biotechnology (Santa Cruz, CA).

Expression vector construction and stable cell lines establishment

RNF13 cDNA was cloned into the pcDNA4.0-Myc-His vector with the forward primer 5'-CCC AAG CTT ATG CTG CTC TCC ATA GGG ATG CTC A-3' and the reverse primer $5^{\prime}$-CCG CTC GGA ACA GTA TTT GCT ATG TTG TAA TCC- ${ }^{\prime}$. For generating C258A/H260A mutant the internal primers 5'-AGA ATC CTT CCC TGT TCC CAT GCT TAT CAT T-3' and 5'-GCAATG ATAAGC ATG GGAACA GGG AAG GAT-3' were used, and for W270A mutant the internal primers were 5'- GGT TTT AGT TAG CCA AGG GTC TAC ACA CTT GCAAT-3' and 5'-GCAAGT GTG TAG ACC CTT GGC TAA CTA AAA CCA A-3', respectively. The nucleotide sequence encoding the HA epitope was introduced into $3^{\prime}$ of human RNF13 cDNA ORF (wild-type and RING domain mutant C258A/H260A) for constructing the $\mathrm{pEAK} 13$ expression vector. $\mathrm{N} 43 \mathrm{~A}$ (primers: 5'-GCA TAT AAC TTT GAA GCT GCA TCT CAG AC-3' and 5'-GTC TGA GAT GCA GCT TCA AAG TTA TAT GC- $3^{\prime}$ ) and N88A (primers: 5'-TCC ACC AGT AAA AGA CGC TTC ATC TGG CAC-3' and 5'GTG CCA GAT GAA GCG TCT TTT ACT GGT GGA-3') mutants were cloned into pEAK13 as the strategy of RING mutants. Stable transformants (MiaP2-vector, MiaP2-RNF13 WT and MiaP2-RNF13 Mutant) were developed by cloning the colonies under selection with $800 \mu \mathrm{g} / \mathrm{mL}$ G418 (Sigma-Aldrich, St Louis, MO).

\section{In vitro and in vivo ubiquitination assay}

One microgram purified His-RNF13 (207-374) recombinant protein was incubated for $1 \mathrm{~h}$ at $30^{\circ} \mathrm{C}$ in a $10 \mu \mathrm{L}$ reaction mixture, consisted of $100 \mathrm{nM}$ E1 (ubiquitin activating enzyme, Sigma-Aldrich), $1 \mu \mathrm{M}$ each E2 (ubiquitin conjugating enzyme set containing $\mathrm{UbcH} 2, \mathrm{UbcH} 3, \mathrm{UbcH} 5 \mathrm{a}, \mathrm{UbcH} 5 b, \mathrm{UbcH} 5 \mathrm{c}, \mathrm{UbcH} 6, \mathrm{UbcH} 7$, and UbcH10 from Calbiochem), $5 \mu \mathrm{M}$ ubiquitin (Sigma-Aldrich), 50 $\mathrm{mM}$ Tris- $\mathrm{HCl}$ (pH 7.5), $2 \mathrm{mM}$ ATP, $2.5 \mathrm{mM} \mathrm{MgCl}_{2}$ and $1 \mathrm{mM}$ DTT. The reaction was stopped by adding $10 \mu \mathrm{L} 2 \times$ SDS loading buffer. The boiled samples were separated on $8 \%$ SDS-PAGE and immuneblotted with the anti-ubiquitin antibody (P4D1).

Ubiquitination assay of wild-type (WT) Myc-RNF13 and its two 
mutants $(\mathrm{C} 258 \mathrm{~A} / \mathrm{H} 260 \mathrm{~A}$ and W270A) expressed in COS-7 cells was performed as described [33]. In brief, immune precipitates from the transfected cells were incubated with $5 \mu \mathrm{M}$ ubiquitin, $100 \mathrm{nM}$ $\mathrm{E} 1,1 \mu \mathrm{M}$ UbcH5a in $50 \mathrm{mM}$ Tris buffer $(\mathrm{pH} 7.5)$ containing $2 \mathrm{mM}$ ATP, $2.5 \mathrm{mM} \mathrm{MgCl}{ }_{2}$ and $1 \mathrm{mM}$ DTT for $1 \mathrm{~h}$ at $30^{\circ} \mathrm{C}$. The mixtures were separated on $8 \%$ SDS-PAGE and immune-blotted with antiubiquitin antibody.

For the in vivo ubiquitination assay, COS-7 cells were transiently co-transfected with either $10 \mu \mathrm{g}$ of Myc-RNF13 (WT) or its mutants (C258A/H260A, W270A) and $5 \mu \mathrm{g}$ HA-ubiquitin, respectively. As controls, cells were transfected by $10 \mu \mathrm{g}$ of vector DNA and $5 \mu \mathrm{g}$ HA-ubiquitin. After $24 \mathrm{~h}$, the cells were treated with $10 \mathrm{mM} \mathrm{MG-}$ 132 for $4 \mathrm{~h}$. The clarified cell lysates were immune precipitated with anti-Myc antibody and protein $\mathrm{A} / \mathrm{G}$ plus agarose and washed with lysis buffer with MG-132 and protease inhibitor cocktail. Samples were separated by $8 \%$ SDS-PAGE and immune-blotted with antiHA antibody.

\section{Patients and specimens}

Human tissue samples were used according to the guidelines of the Ethical Committee of the Peking Union Medical College Hospital. Surgically resected specimens from 72 patients (age range, 29-78 years) with PDAC were examined. The patient population represents a randomly selected subgroup from a clinical series that includes all patients undergoing surgical resection between June 1998 and December 2005 in the Department of Surgery at Peking Union Medical College Hospital. The diagnosis of PDAC, histological grading and pathologic stage were re-evaluated and/or confirmed by an independent pathologist. PanIN lesions $(n=35)$ and CP $(n=29)$ were assessed and graded in pancreatic tissues adjacent to the cancer in hematoxylin and eosin-stained slides. PDAC tissue array containing 79 independent PDAC and 3 normal pancreatic tissue samples were purchased from Cybrdi Inc., ShanXi, China.

\section{Immunohistochemistry}

Immunostaining for RNF13 was performed using the primary goat polyclonal antibody against human RNF13 (diluted 1:150) overnight after antigen retrieval in $10 \mathrm{mM}$ sodium citrate buffer $(\mathrm{pH}$ 6.0) for $15 \mathrm{~min}$ at $95^{\circ} \mathrm{C}$, followed by incubation with HRP-labeled anti-goat antibody for $1 \mathrm{~h}$. Tenascin-C staining was performed after antigen retrieval of consecutive sections from samples used for RNF13 staining pretreated with $0.5 \mathrm{mg} / \mathrm{mL}$ Pronase at $37{ }^{\circ} \mathrm{C}$ for $15 \mathrm{~min}$. The sections were then incubated with mouse monoclonal anti-Tenascin-C antibody overnight at $4{ }^{\circ} \mathrm{C}$. Immunostaining and clinicopathologic features were evaluated microscopically by two pathologists (Jie Chen and Yun-Xiao Meng).

RNF13-specific immunoreactivity was scored by estimating the percentage of labeled tumor cells as follows: score $0,<25 \%$ positive cancer cells; score,$+ 25-50 \%$ positive cancer cells; score ++ , $50-75 \%$ positive cancer cells; score,$+++>75 \%$ positive cancer cells. Specimens were considered positive for RNF13 expression when the scores were + to +++ , and were considered negative for RNF13 expression when the score was 0 . Pictures were collected with $\mathrm{Mi}$ croView MVC2000 image apparatus and software.

\section{Statistical analysis}

Data are expressed as mean $\pm \mathrm{SD}$. Comparisons between groups were done using Welch's $t$ test. Correlation of RNF13 immunoreactivity with clinicopathologic parameters and Tenascin-C expression were analyzed by Fisher's exact test. Differences were considered significant at $P<0.05$.

\section{3-(4,5-Dimethylthiazol-2-yl)-2,5-diphenyltetrazolium bromide (MTT) assay}

Cells were seeded in 96-well plates $(n=4)$ and grown for 1, 3, 5 days, followed by the MTT addition. MTT reduction into insoluble formazan was measured at $570 \mathrm{~nm}$ after the purple formazan crystals were dissolved in solution containing $10 \%$ SDS and $0.01 \mathrm{M} \mathrm{HCl}$.

\section{Matrigel invasion assay}

A $10 \mu \mathrm{L}$ aliquot of Matrigel $(10 \mathrm{mg} / \mathrm{mL})$ was spread onto a 6.5 $\mathrm{mm}$ Transwell filter with $8 \mu \mathrm{m}$ pores (Costar, Corning, NY). The lower chamber of the Transwell contained serum-containing (10\%) media. Cells were suspended in serum-free media with $0.1 \%$ BSA $\left(5 \times 10^{4}\right.$ cells $\left./ \mathrm{mL}\right)$, and $0.5 \mathrm{~mL}$ of cell suspension was added to the top chamber and incubated for $48 \mathrm{~h}$. The cell suspension was aspirated, and excess Matrigel was removed from the filter using a cotton swab. The fixed filters were stained with Crystal Violet and the cells in 5 random $\times 40$ magnification fields were counted.

\section{Gelatin zymography}

Equal numbers of cells from the MiaP2-vector, MiaP2-RNF13 WT and MiaP2-RNF13 Mutant were plated and allowed to reach confluence over $24 \mathrm{~h}$, respectively. Cells were then incubated for $24 \mathrm{~h}$ in $1.5 \mathrm{~mL}$ of serum-free DMEM. Conditioned media were collected, concentrated and analyzed with 10\% SDS-PAGE co-polymerized with $0.25 \mathrm{mg} / \mathrm{mL}$ of gelatin under non-reducing conditions. Gels were washed in $2.5 \%$ Triton $\mathrm{X}-100$ for $30 \mathrm{~min}$, and incubated in collagenase buffer $\left(100 \mathrm{mmol} / \mathrm{L}\right.$ Tris- $\mathrm{HCl} \mathrm{pH} 8.0,5 \mathrm{mmol} / \mathrm{L} \mathrm{CaCl}_{2}$, $0.02 \% \mathrm{NaN}_{3}$ ) for $40 \mathrm{~h}$ at $37{ }^{\circ} \mathrm{C}$. Gels were stained for $30 \mathrm{~min}$ with $0.5 \%$ coomassie blue and then de-stained for $1 \mathrm{~h}$.

\section{Acknowledgments}

This study was supported by the following grants: National Basic Research Program of China grants 2005CB522405, 2005CB522507, 2006CB943503 and 2007CB946903; the National Natural Science Foundation of China grants 30570984, 30721063. We thank Dr Chengyu Jiang (Institute of Basic Medical Sciences, Chinese Academy of Medical Sciences) for providing the pEAK13 plasmid. We also thank Dr Francesco M Marincola (Department of Transfusion Medicine, Clinical Center, National Institutes of Health) for critical reading of this manuscript and Dr Limin Li (Institute of Basic Medical Sciences, Chinese Academy of Medical Sciences) for comments on manuscript preparation.

\section{References}

1 Burger A, Amemiya Y, Kitching R, Seth AK. Novel RING E3 ubiquitin ligases in breast cancer. Neoplasia 2006; 8:689-695.

2 Subramaniam V, Li H, Wong M, et al. The RING-H2 protein RNF11 is overexpressed in breast cancer and is a target of Smurf2 E3 ligase. Br J Cancer 2003; 89:1538-1544.

3 Kitching R, Wong MJ, Koehler D, et al. The RING-H2 protein RNF11 is differentially expressed in breast tumours and interacts with HECT-type E3 ligases. Biochim Biophys Acta 2003; 1639:104-112.

4 Burger AM, Gao Y, Amemiya Y, et al. A novel RING-type 
ubiquitin ligase breast cancer-associated gene 2 correlates with outcome in invasive breast cancer. Cancer Res 2005; 65:1040110412 .

5 Wan M, Huang J, Jhala NC, et al. SCF(beta-TrCP1) controls Smad4 protein stability in pancreatic cancer cells. Am J Pathol 2005; 166:1379-1392.

6 Calhoun ES, Jones JB, Ashfaq R, et al. BRAF and FBXW7 (CDC4, FBW7, AGO, SEL10) mutations in distinct subsets of pancreatic cancer: potential therapeutic targets. Am J Pathol 2003; 163:1255-1260.

7 Jemal A, Siegel R, Ward E, et al. Cancer statistics, 2007. CA Cancer J Clin 2007; 57:43-66.

8 Hruban RH, Adsay NV, Albores-Saavedra J, et al. Pancreatic intraepithelial neoplasia: a new nomenclature and classification system for pancreatic duct lesions. Am J Surg Pathol 2001; 25:579-586.

9 Tranque P, Crossin KL, Cirelli C, Edelman GM, Mauro VP. Identification and characterization of a RING zinc finger gene (C-RZF) expressed in chicken embryo cells. Proc Natl Acad Sci USA 1996; 93:3105-3109.

10 Juuti A, Nordling S, Louhimo J, Lundin J, Haglund C. Tenascin $\mathrm{C}$ expression is upregulated in pancreatic cancer and correlates with differentiation. J Clin Pathol 2004; 57:1151-1155.

11 Jahkola T, Toivonen T, Nordling S, von Smitten K, Virtanen I. Expression of tenascin-C in intraductal carcinoma of human breast: relationship to invasion. Eur J Cancer 1998; 34:16871692.

12 Adams M, Jones JL, Walker RA, Pringle JH, Bell SC. Changes in tenascin-C isoform expression in invasive and preinvasive breast disease. Cancer Res 2002; 62:3289-3297.

13 Leins A, Riva P, Lindstedt R, et al. Expression of tenascin-C in various human brain tumors and its relevance for survival in patients with astrocytoma. Cancer 2003; 98:2430-2439.

14 Loukopoulos P, Shibata T, Katoh H, et al. Genome-wide arraybased comparative genomic hybridization analysis of pancreatic adenocarcinoma: identification of genetic indicators that predict patient outcome. Cancer Sci 2007; 98:392-400.

15 Mueller DL. E3 ubiquitin ligases as T cell anergy factors. Nat Immunol 2004; 5:883-890.

16 Anandasabapathy N, Ford GS, Bloom D, et al. GRAIL: an E3 ubiquitin ligase that inhibits cytokine gene transcription is expressed in anergic CD4+ T cells. Immunity 2003; 18:535-547.

17 Yamada HY, Gorbsky GJ. Tumor suppressor candidate TSSC5 is regulated by UbcH6 and a novel ubiquitin ligase RING105. Oncogene 2006; 25:1330-1339.

18 Gmachl M, Gieffers C, Podtelejnikov AV, Mann M, Peters JM.
The RING-H2 finger protein APC11 and the E2 enzyme UBC4 are sufficient to ubiquitinate substrates of the anaphase-promoting complex. Proc Natl Acad Sci USA 2000; 97:8973-8978.

19 Joazeiro CA, Wing SS, Huang H, et al. The tyrosine kinase negative regulator c-Cbl as a RING-type, E2-dependent ubiquitin-protein ligase. Science 1999; 286:309-312.

20 Orend G, Chiquet-Ehrismann R. Tenascin-C induced signaling in cancer. Cancer Lett 2006; 244:143-163.

21 Esposito I, Penzel R, Chaib-Harrireche M, et al. Tenascin C and annexin II expression in the process of pancreatic carcinogenesis. $J$ Pathol 2006; 208:673-685.

22 Mahon P, Bateman A. The PA domain: a protease-associated domain. Protein Sci 2000; 9:1930-1934.

23 Luo X, Hofmann K. The protease-associated domain: a homology domain associated with multiple classes of proteases. Trends Biochem Sci 2001; 26:147-148.

24 Joazeiro CA, Weissman AM. RING finger proteins: mediators of ubiquitin ligase activity. Cell 2000; 102:549-552.

25 Asomaning K, Reid AE, Zhou W, et al. MDM2 promoter polymorphism and pancreatic cancer risk and prognosis. Clin Cancer Res 2008; 14:4010-4015.

26 Ruiz C, Huang W, Hegi ME, et al. Growth promoting signaling by tenascin-C [corrected]. Cancer Res 2004; 64:7377-7385.

27 Fryer BH, Field J. Rho, Rac, Pak and angiogenesis: old roles and newly identified responsibilities in endothelial cells. Cancer Lett 2005; 229:13-23.

28 Zhu L, Shi G, Schmidt CM, Hruban RH, Konieczny SF. Acinar cells contribute to the molecular heterogeneity of pancreatic intraepithelial neoplasia. Am J Pathol 2007; 171:263-273.

29 Zhang L, Sanderson SO, Lloyd RV, Smyrk TC. Pancreatic intraepithelial neoplasia in heterotopic pancreas: evidence for the progression model of pancreatic ductal adenocarcinoma. Am J Surg Pathol 2007; 31:1191-1195.

30 Hruban RH, Fukushima N. Pancreatic adenocarcinoma: update on the surgical pathology of carcinomas of ductal origin and PanINs. Mod Pathol 2007; 20:S61-S70.

31 Rosty C, Geradts J, Sato N, et al. p16 Inactivation in pancreatic intraepithelial neoplasias (PanINs) arising in patients with chronic pancreatitis. Am J Surg Pathol 2003; 27:1495-1501.

32 Deryugina EI, Quigley JP. Matrix metalloproteinases and tumor metastasis. Cancer Metastasis Rev 2006; 25:9-34.

33 Furukawa M, Ohta T, Xiong Y. Activation of UBC5 ubiquitinconjugating enzyme by the RING finger of ROC1 and assembly of active ubiquitin ligases by all cullins. J Biol Chem 2002; 277:15758-15765.

(Supplementary information is linked to the online version of the paper on the Cell Research website.) 